\title{
Reestructuración, crecimiento y expansión metropolitana en las economías emergentes latinoamericanas*
}

\author{
Carlos A. de Mattos \\ Instituto de Estudios Urbanos \\ Pontificia Universidad Católica de Chile
}

\section{Resumen}

Contradiciendo algunas previsiones hechas cuando comenzaron a apreciarse los primeros impactos territoriales de los procesos de reestructuración, desencadenados como respuesta a la crisis del fordismo, durante los últimos años, al acentuarse la globalización, se ha comprobado una generalizada recuperación del crecimiento y la expansión de las principales áreas metropolitanas de economías maduras y emergentes. Esta tendencia aparece asociada a la intensificación de la movilidad de un capital, crecientemente autonomizado, que muestra una clara preferencia por aquellos lugares cuya historia productiva los ha dotado de un mayor potencial endógeno $y$, por ende, de una mayor fertilidad productiva. Vale decir, que son los lugares que ofrecen una mayor acumulación inicial de capital físico, capital humano y conocimientos, los que muestran también una mayor atractividad vis-a-vis de los movimientos internacionales e interregionales del capital. En especial, se ha comprobado que ello incide fuertemente en los factores de localización que privilegian tanto el comando del nuevo poder económico, como los servicios avanzados y la parte más innovadora y dinámica de 
la industria posfordista. Distintas investigaciones muestran que esta tendencia ya se observa en la mayoría de las economías emergentes latinoamericanas. De esta forma, las áreas metropolitanas heredadas del periodo precedente del desarrollo capitalista está dando paso a metrópolis-regiones, que en su persistente expansión se van configurando como verdaderos archipiélagos urbanos de fronteras difusas, signando un tipo de configuración territorial en la que coexisten con grandes espacios vacíos o semivacíos.

\section{Introducción}

El derrumbe de los experimentos de planificación centralizada, la crisis fiscal del Estado de bienestar y el agotamiento del régimen de acumulación fordista, el progresivo abandono de las estrategias macroeconómicas de corte keynesiano, la afirmación de un nuevo paradigma científico-técnico articulado en torno a las nuevas tecnologías de la información, el incontenible avance del proceso de mundialización de las distintas formas del ciclo del capital y la generalización de estrategias de reestructuración basadas en una radical liberalización económica, aparecen como los hitos centrales de los profundos cambios que han afectado al mundo entero durante las últimas dos décadas. Todo esto marca la transición hacia una nueva fase de la evolución del capitalismo en la que se perfila un nuevo escenario, cuyos rasgos sociales, económicos, políticos, culturales, territoriales, etc., presentan diferencias substanciales con respecto alos que habían caracterizado la fase precedente.

Desde que estas transformaciones comenzaron a esbozarse, numerosos estudios plantearon que con ellas se habría iniciado el camino hacia una efectiva reversión de la polarización y, por lo tanto, hacia una mayor dispersión territorial de los focos de acumulación y de crecimiento $y$, aun, que esto constituía la primera etapa de un proceso que habría de llevar hacia una mayor convergencia de los ingresos per cápita internacionales e inter-regionales. Estos planteamientos nacieron y se desarrollaron respaldados, por una parte, por las previsiones de una corriente teórico-ideológica que ganó rápidamente predicamento en ese momento $y$, por otra parte, por las conclusiones de algunos estudios realizados cuando la crisis del

fordismo aún no había concluido y los procesos de reestructuración todavía se encontraban en sus etapas iniciales.

Sin duda, la fuerte influencia de los modelos de corte neoclásico en boga, especialmente, durante los primeros años de la aplicación de las políticas de liberalización económica y de desregulación, llevaron a dar por ciertas las previsiones de que una mayor libertad en el juego de las fuerzas del mercado, propiciaría una mayor convergencia internacional e inter-regional lo que, con el andar del tiempo, se transformó en una suerte de profecía autocumplida. Pero, además, estas previsiones fueron reforzadas por los resultados de algunos estudios empíricos realizados en países donde al inicio de los procesos de reestructuración, efectivamente, se observó - y todavía se observa - un cierto despliegue territorial productivo-demográfico, lo que dio pie para que se tendiese a concluir prematuramente, que este tipo de comportamiento podía considerarse como un rasgo inherente a la nueva dinámica económica. Ello suponía asumir que lo que se estaba observando en la etapa inicial de la transición entre dos fases del desarrollo capitalista podía interpretarse como una tendencia permanente.

El que cierta dispersión territorial haya seguido manifestándose en el interior de algunos países periféricos, que se encuentran a medio camino en sus procesos de reestructuración e inserción competitiva en la economía-mundo, ha dado pie para que se continúe insistiendo en que se está en camino hacia una mayor convergencia internacional e inter-regional. Al hacerlo, se tiende a soslayar que durante los periodos de recesión o de crisis económica, generalmente se observa una mayor dispersión territorial de las actividades económicas y de la población y, en consecuencia, una cierta atenuación de las desigualdades inter-regionales del ingreso per cápita, en tanto que en los periodos de recuperación del crecimiento económico, este comportamiento muestra habitualmente indicios de reversión.

Además, las tendencias observables en los países que han mostrado mayores avances en su reestructuración e inserción competitiva en la economía-mundo, no parecen avalar tales conclusiones. En efecto, en la medida que los rendimientos crecientes y la competencia oligopólica han ido afirmando con más fuerza su presencia en el nuevo régimen de acumulación, la divergencia en los ritmos de crecimiento entre países y entre regiones parece haber retomado su carácter de rasgo congénito a la propia dinámica capitalista. De hecho, 
un conjunto de regularidades empíricamente comprobables, ${ }^{1}$ así como los resultados de diversas investigaciones realizadas para distintos páses desarrollados, han suministrado elementos de juicio para cuestionar las previsiones de convergencia. Lo que es sintetizado drásticamente por Krugman (1992: 11), cuando afirma que la respuesta más breve a la pregunta sobre cuál sería la característica más prominente de la distribución geográfica de la actividad económica sería que "es seguramente su concentración".

Desde esta perspectiva, también es posible poner en tela de juicio los augurios sobre reversión de la polarización, de la crisis de las grandes ciudades y del crecimiento metropolitano. A este respecto, por ejemplo, Veltz (1996:22), luego de analizar casos como los de Francia, Inglaterra y Japón, concluye que “[...] la 'metropolización' de la economía se afirma como la tendencia principal del decenio". Por su parte, Chinitz y Moran (1996: 1), al estudiar las tendencias observadas en el periodo 1980-1990 en los Estados Unidos, observan que "dentro de todas las regiones, la tendencia ha sido siempre hacia las mayores aglomeraciones metropolitanas". En el mismo sentido, las conclusiones de diversos estudios de carácter prospectivo sobre caminos alternativos, realizados considerando las tendencias desencadenadas a partir de la crisis del fordismo, apuntan en esta dirección. ${ }^{2}$

De esta manera, luego de algunos años de relativo optimismo, en los que se creyó que las nuevas modalidades de organización y distribución de la producción llevarían a una mayor convergencia inter-regional y al estancamiento del crecimiento metropolitano, la intensificación de la concentración productivo-demográfica, en un número limitado de lugares, ha vuelto a presentarse como una tendencia predominante en el mapa mundial, aun cuando con ciertas

'Así, por ejemplo, en la evolución de las últimas décadas se ha podido comprobar que "[...] se mida como se mida, la distribución de las capacidades innovadoras entre los diferentes páses es altamente desigual. El número de participantes en el 'club de innovadores' es bastante pequeño y relativamente estable a lo largo del tiempo" (Dossi, 1991: 170). En la misma dirección, Pavitt y Soete señalan que "los crecimientos en los niveles de productividad de los países en las décadas de 1960 y 1970 con respecto a la media mundial, estuvieron asociados a incrementos en las actividades innovadoras, medidas en términos de gasto en $\mathrm{I}+\mathrm{D}$ y de registro de patentes en el extranjero" (citado por Dossi, 1991: 171). Ninguna de estas tendencias permiten avizorar un camino de convergencia.

${ }^{2}$ Tal es el caso, por ejemplo, de un ejercicio de prospectiva espacial realizado por Lipietz y Leborgne (1989: 132), en el que luego de analizar tres posibles modelos de organización del trabajo, concluyen que "[...] todos estos modelos tienen en común una tendencia a privilegiar las aglomeraciones urbanas, lo que es resultado del reforzamiento del carácter mercantil en las relacines antre amnresas a través de la cuasi integración vertical". particularidades se establecen diferencias con lo que se observaba en el periodo fordista.

Frente a esta situación, este trabajo se propone analizar las tendencias dominantes en la distribución territorial de las actividades productivas y de la población, con particular referencia al caso de los países latinoamericanos que más han avanzado en sus procesos de reestructuración y modernización y, por ende, en la consolidación de la urbanización de sus economías. A estos efectos, se observará en primer lugar cual ha sido la orientación predominante de los movimientos internacionales e inter-regionales del capital a medida que se han ido intensificando los procesos de modernización capitalista asociados a la globalización. En segundo lugar, se estudiará cuáles son los lugares y los sectores preferidos para la materialización de esos flujos, teniendo presente, especialmente, sus efectos en términos de acumulación nacional, regional y/o local. A partir de allí, se buscará identificar los factores que inciden con más fuerza en las decisiones sobre localización de las actividades productivas, en especial las más modernas y dinámicas, mostrando la vinculación de estos factores con las condiciones inherentes a las grandes aglomeraciones metropolitanas. En este contexto, se mostrará cómo ello influye en la formación y expansión de los correspondientes mercados de trabajo $y$, por consiguiente, en la distribución territorial de la población y en la recuperación del crecimiento metropolitano. Finalmente, con estos antecedentes, se analizarán las nuevas tendencias a la concentración urbana y la expresión territorial emergente de la expansión productivo-demográfica metropolitana, considerando en especial sudinámica social interna.

\section{Globalización, liberalización, desregulación y movimientos del capital}

Los cambios observados a medida que países y empresas fueron saliendo de la crisis que culminó hacia mediados de la década de los años setenta, producidos bajo el impulso simultáneo e interrelacionado de la consolidación de un nuevo paradigma científico-técnico y la profundización de la globalización del capital, estuvieron marcados por el ascendente predicamento de la idea de que la posibilidad de acumular y crecer en el ámbito de la nueva dinámica económica, se encontraba condicionada por la capacidad, tanto de las naciones como de las empresas, para aumentar su competitividad. $\mathrm{Al}$ aceptar que esto constituía una exigencia ineludible para la supervivencia en el nuevo 
escenario, un número creciente de países optó por impulsar cambios radicales en la orientación de sus estrategias y políticas macroeconómicas, en el entendido de que las condiciones generales para mejorar la competitividad, deberían ser establecidas ab initio en el ámbito nacional. ${ }^{3}$

Fue así como en el ámbito de la gestión pública nacional, comenzó a ser impulsado un profundo proceso de reestructuración inspirado en una concepción teórico-ideológica, que se sustentaba en la certeza de que al "soltar a las fuerzas del mercado" - que bajo el apogeo de las ideas keynesianas habían sido fuertemente constreñidas durante varias décadas - sería posible mejorar las condiciones para la valorización privada del capital. En esencia, se suponía que una radical liberalización económica era el camino idóneo para restablecer las condiciones necesarias para dinamizar los procesos de acumulación y crecimiento. A su vez, en un ambiente signado por el aumento de la incertidumbre y los riesgos, las empresas se vieron compelidas a realizar cambios sustanciales en la organización de sus procesos productivos, a fin de modificar las bases estructurales de su competitividad, a manera de poder ganar espacio en la economía globalizada $\mathrm{y}$, de esta forma, asegurar su crecimiento y supervivencia.

En este escenario, la desregulación, componente básico de las estrategias de liberalización económica, se hasituado como un proceso complementario a la globalización del capital. De hecho, se trata de procesos que se retroalimentan recíprocamente $y$, al hacerlo, contribuyen a profundizar la nueva dinámica económica: mientras la globalización requiera de la mayor liberalización y apertura en el funcionamiento delas distintas economías nacionales, la desregulación se ubica como condición ineludible para que una economía nacional pueda mejorar su inserción en la dinámica globalizada. De la aplicación de esta estrategia ha emergido un conjunto de transformaciones estructurales de las que, para el análisis que se propone realizar en este trabajo, es importante destacar:

${ }^{3}$ Una importante conclusión de la investigación de Porter sobre las ventajas competitivas de las naciones es que "las diferencias a escala nacional en estructuras económicas, valores, culturas, instituciones e historias contribuyen profundamente al éxito competitivo. El papel de la nación parece ser tan fuerte como antes o incluso más fuerte que nunca" (Porter, 1990: 45). Esta conclusión contradice abiertamente las tesis de algunos autores, como por ejemplo la de Ohmae (1995), en el sentido de que se estaría evolucionando hacia la desaparición del Estado-nación. La observación sobre el aumento de la competitividad de las empresas de los países que más han avanzado en sus procesos de reestructuración parecen avalar decisivamente la conclusión de Porter. a) La creciente autonomización del capital, tanto en lo que respecta a las indicaciones públicas, como a sus relaciones con lo material, que surge como una consecuencia natural del despliegue de las nuevas tecnologías de la información y las condiciones generales establecidas por la desregulación. ${ }^{4}$ Ello se manifiesta en una progresiva pérdida de la capacidad de los Estados nacionales para la gestión de los movimientos del capital que, cada día con mayor fuerza, tienden a sobrepasar las regulaciones y las fronteras nacionales, en pos de los destinos sectoriales o territoriales percibidos como más rentables. Al mismo tiempo, como expresión del mismo proceso, el mercado del dinero, paulatinamente, se ha ido divorciando del de las mercancías, de tal manera que el flujo de capitales ha ido perdiendo relación con el volumen del comercio internacional, en una secuencia de progresiva autonomización del componente financiero (Menzel, 1995: 8-12). Por su parte, esta autonomización conlleva a dos epifenómenos adicionales que habrán de tener una importante incidencia en la distribución territorial de las actividades productivas:

- la intensificación de la secular tendencia a la conglomeración del capital, en un cuadro donde las fusiones, adquisiciones, "joint-ventures", etc., han adquirido el carácter de hechos cotidianos en el noticiero de la economía mundial, ${ }^{5}$ con el obvio resultado de que un número creciente de empresas (imbricadas en redes globales, estimuladas por las condiciones establecidas por las políticas de liberalización y desregulación), se han afirmado como las protagonistas indiscutidas de la nueva dinámica económica. En consecuencia, las perspectivas en materia de acumulación y crecimiento para las naciones o las regiones dependen, cada día en mayor grado, de las estrategias y de las decisiones de unas

4 En este sentido, Lafay (1996: 51-52) afirma que "los movimientos internacionales del capital constituyen la manifestación más espectacular del proceso de mundialización. Ellos han sido acelerados tanto por las decisiones de los Estados (la desregulación), como por las mutaciones tecnológicas (permitiendo, a bajo costo, una difusión instantánea de las informaciones)".

${ }^{5}$ Como señala Coriat, "las grandes firmas ya en parte internacionalizadas, han debido modificar su dimensión, participando de operaciones muy importantes de fusión y adquisición, de compra de otras empresas y de tomas de participación financiera para poder estar presentes en otros mercados" (Coriat, 1997: 18). 
estructuras empresariales organizadas jerárquicamente a escala mundial;

el progresivo debilitamiento de las raíces territoriales del capital, consecuencia lógica de la formación, expansión y consolidación de dichas estructuras empresariales multinacionales y multi-regionales, cuya presencia en la cúpula de los procesos decisivos internacionales y nacionales de acumulación, determina la dirección y el destino de un capital que tiende a moverse, en una incesante búsqueda de los sectores y territorios de mayor rentabilidad, minimizando las consideraciones sobresusorígenes o susvinculaciones nacionales, regionales o locales.

b) La paulatina transformación de la morfología organizativa de las empresas, derivada de la necesidad de aumentar su competitividad en el escenario globalizado, las obliga a buscar formas de organización que permitan una mayor flexibilidad para enfrentar los continuos cambios del mercado. En una situación, en la que se ha ido imponiendo una transición desde una competencia vía costos hacia una competencia a través de la diferenciación de las mercancías (Veltz, 1996), la organización en red se ha constituido en un tipo de respuesta habitual. A este respecto, Lafay (1996: 49) señala que la empresa-red constituye la forma más reciente de la internacionalización, destaca que "en lugar de crear filiales estrictamente controladas en el seno de un sistema fuertemente estructurado y jerarquizado, se hace más rentable establecer relaciones contractuales con las contrapartes que emergen en los países en que se implantan, especialmente en los países en despegue industrial". Con ello, se ha generalizado la desintegración vertical de un número creciente de grandes empresas, donde se hacen cada día más frecuentes los procesos de externalización de procesos y actividades y su posterior reconstitución a través de formas de "cuasi integración vertical" (Lipietz y Leborgne, 1988). Con frecuencia, la reconstitución vertical de los procesos productivos por esta vía, tiene carácter transitorio o coyuntural, dando origen al tipo de organización que ha sido bautizada como corporación virtual (Davidow y Malone, 1992). c) La intensificaciónde la terciarización del aparato productivo, ${ }^{6}$ derivada de un fuerte crecimiento mostrado durante las últimas décadas, tanto por los servicios a la población (comercio, salud, etc.), los servicios sociales (cultura, educación, etc.) y el turismo; como por los servicios a la producción, que ha redundado en que este sector genere entre 60 y $70 \%$ del total del empleo en los países que más han avanzado en su modernización capitalista. En este proceso, se observan importantes cambios cualitativos en la composición interna del sector, consecuencia fundamentalmente del impacto de la microelectrónica y de las nuevas tecnologías de la información en la organización de los procesos productivos y donde buena parte de las grandes empresas industriales han tendido a externalizar un elevado número de actividades que, desde entonces, han pasado a ser contabilizadas como servicios. De hecho, este crecimiento de la participación del sector terciario, en la generación de empleo, ha incidido decisivamente también en un mayor crecimiento de los mercados urbanos de trabajo.

Este conjunto de cambios se reflejó en una fuerte acentuación -hasta cierto punto, culminación-delos procesos de desruralización de la economía, procesos que, en el caso de los países latinoamericanos más industrializados, habían comenzado a acelerarse rápidamente en la fase de desarrollo hacia adentro, en especial bajo los efectos de la industrialización sustitutiva. Como consecuencia de ello, en todos estos países se observa una significativa cá́da de la contribución de la agricultura al PIB y, al mismo tiempo, un aumento paulatino y sostenido de la tasa de urbanización. Todo esto tiene importantes repercusiones en la distribución territorial de los focos de acumulación y crecimiento.

\section{Movimientos del capital y atractividad territorial}

Para identificar los principales impactos territoriales de la nueva dinámica económica, emergente de las transformaciones señaladas, es necesario precisar ante todo, hacia donde se dirigen preferentemente esos capitales que ahora se mueven prácticamente sin restricciones $y$,

${ }^{6}$ Ferrao destaca que "[...] a partir del momento en que se pasa a entender el proceso de terciarización como un componente central de los mecanismos de reestructuración de los sistemas productivos y socio-culturales y no apenas como una extensión del denominado 'sector terciario', terciarización, innovación y modernidad surgen ineluctablemente asociadas' (1992: 35). 
en seguida, establecer cuáles son los atributos que explican por qué ellos se materializan en mayor grado en determinados lugares y no en otros. En lo que respecta al destino territorial de un capital crecientemente autonomizado, conglomerado y desarraigado, la evidencia empírica disponible es concluyente en mostrar que el mismo no se dirige hacia los lugares más desregulados y con salarios más bajos, sino hacia los territorios percibidos como menos riesgosos y más rentables, ${ }^{7}$ o que posean una mayor fertilidad relativa según la proposición de Kampetter (1995), independientemente de si allí los salarios son más altos y las regulaciones más duras. Vale decir, al amparo de las condiciones establecidas por las políticas de liberalización y desregulación, el capital tiende a dirigirse, preferentemente, hacia los sectores, actividades y lugares que le resultan más atractivos, en función de las posibilidades que allí encuentra para germinar más rápida $\mathrm{y}$ vigorosamente.

¿De qué depende la atractividad de un territorio? En lo esencial, podría decirse que esta condición depende de la presencia (o ausencia) de un conjunto de atributos configurados a lo largo de la específica historia productiva de cada territorio. A este respecto, Marconnet y Fremeaux (1996: 70) precisan que "la atractividad de un territorio depende de múltiples factores: costo y calidad de la mano de obra, pero también de la calidad del tejido industrial local (para la subcontratación), de la calidad de las infraestructuras de transportes y comunicaciones, así como de la apertura del mercado de capitales y de las ayudas políticas a la inversión". Coincidentemente, las explicaciones aportadas por las nuevas teorías del crecimiento (Romer, Lucas, Barro, etc.) indican que los lugares de más elevado potencial endógeno, serían aquellos que cuentan con una mayor acumulación inicial de capital físico, capital humano y conocimientos (Guellec y Ralle, 1995). Sin duda, el hecho de que las evaluaciones de riesgo-país y de competitividad consideren habitualmente, atributos de esta naturaleza para elaborar las tablas respectivas, suministra un respaldo empírico relevante acerca de que los propietarios del capital, en efecto, lés otorgan crucial importancia cuando adoptan sus decisiones acerca de

7 La información disponible para los últimos 25 años, muestra que esta tendencia se ha acentuado a medida que el proceso de globalización se ha ido intensificando. Al respecto, Hatem (1995: 84) destaca que "el impulso de los flujos de IED a partir de 1985 han beneficiado sobre todo a los países desarrollados. Estos han recibido, entre 1985 y 1990, 85\% de los flujos acumulados, contra solamente $72 \%$ entre 1980 y $1984^{\circ}$ donde invertir. ${ }^{8}$ Tanto a escala internacional como nacional, su mayor o menor presencia tiene un papel determinante en las decisiones de los propietarios del capital sobre localización y/o deslocalización de sus inversiones y condiciona las perspectivas de acumulación y de crecimiento en ese territorio.

Es posible concluir, por tanto, que la situación inicial de cada territorio, configurada por la consolidación de atributos como los señalados, juega un papel decisivo en la distribución territorial -tanto en el plano internacional, como en el interior de cada espacio nacionalde las actividades productivas y, por ende, de los respectivos mercados de trabajo. En otras palabras, sería la incidencia mancomunada de estos atributos, lo que signaría las posibilidades, en cuanto a ritmos de acumulación y crecimiento de cada país y de cada región, en el nuevo escenario globalizado.

La propia lógica inherente a esta dinámica desencadena procesos de carácter acumulativo, desde que los territorios dotados de mayor nivel relativo inicial de stocks de capital físico, capital humano y conocimientos, dadas las externalidades positivas de estos factores, se constituyen en focos de atracción para las nuevas inversiones, logrando de esta forma aumentar dichos stocks de periodo en periodo. Allí, donde esta dinámica de retroalimentación comienza a operar, las tendencias a la concentración y a la divergencia no muestran síntomas relevantes de reversión.

Esta dinámica no afecta por igual a todas las áreas de concentración productivo-demográfica, que al despuntar el proceso de cambio posfordista partieron con una mejor situación inicial; algunas de ellas han tendido al estancamiento y/o a la regresión, siendo desplazadas por otras que lograron mantener, incrementar o recuperar su dinamismo. Sin embargo, un hecho empíricamente comprobable es que las áreas que en esta nueva fase continuaron expandiéndose, ya eran importantes focos de concentración productiva hacia el final del periodo anterior.

En este proceso, se han ido estableciendo ventajas para aquellos territorios nacionales o subnacionales que a lo largo de su historia productiva, han logrado mayores avances en su reestructuración y modernización y, en consecuencia, conformaran más tempranamente

${ }^{8}$ Así, por ejemplo, puede mencionarse que Standard \& Poor evalúa la situación de cada país en función de su riesgo político (sistema político, ambiente social, relaciones internacionales) y del riesgo económico (posición financiera externa, flexibilidad de la balanza de pagos, estructura y crecimiento económico, conducción económica, perspectivas económicas) (Standard \& Poor, Emerging Markets, marzo 1995). 
un escenario macroeconómico atractivo. En esa situación, la desigual dotación inicial de los atributos que caracterizan la atractividad de cada lugar, configura la base sobre la que se reproducen estructuras territoriales jerarquizadas, dibujando un mapa (internacional, interregional e intrarregional) signado por el crecimiento divergente, donde la concentración y la desigualdad se ubican como rasgos congénitos. A partir de esta aproximación de carácter general, es posible intentar ahora una identificación más precisa de los lugares concretos (esos territorios de mayor atractividad relativa) en que tienden a materializarse dichos flujos de capital. A estos efectos, es necesario establecer cuáles son los sectores y tipos de actividad preferidos y, a partir de allí, identificar que factores específicos de localización son privilegiados por estos sectores y actividades al momento de decidir el destino territorial de sus inversiones.

\section{Tendencias locacionales y nuevas modalidades de expansión metropolitana}

¿Hacia que tipo de actividades se dirigen predominantemente los flujos de inversión? Allí donde la reestructuración de las economías nacionales y de las empresas ha logrado avances significativos, los flujos internacionales de inversión extranjera directa (IED) tienden a orientarse preferentemente hacia la industria y, en especial, hacia los servicios, ${ }^{9}$ es ahí donde aparecen en un lugar cada día más destacado los servicios a la producción (bancarios y financieros, seguros, servicios inmobiliarios, ingeniería y arquitectura, servicios contables y legales, así como otros servicios profesionales). La información disponible muestra que la inversión nacional parece seguir patrones de localización análogos a los de la IED, particularmente la que se realiza por las empresas más integradas a la dinámica de la globalización,

9 En el caso de los paises latinoamericanos esta tendencia aparece en forma clara. En un estudio de la CEPAL sobre el tema, se señala que "el aumento del IED en el sector servicios y su caída en el sector manufacturero durante la década de 1980 se tradujo en un incremento de la participación relativa del acervo de IED acumulado en el sector terciario. La IFD en servicios tales como comercio, transportes y comunicaciones surgió inicialmente con carácter de apoyo a las actividades de las ETN's en el sector manufacturero. Las reformas económicas introducidas en la región significaron la eliminación de las restricciones a la IED en algunas actividades de servicios, particularmente bienes raíces, finanzas y seguros, lo que favoreció el ingreso de capitales extranjeros en este sector. Los programas de privatización fueron otro factor importante que explica el crecimiento registrado por la EED en las. actividades de servicios durante los años noventa." (CEPAL, 1995: 13). tendencia que se observa tanto en los países desarrollados como en las economías emergentes.

Para identificar los impactóos de la materialización territorial de estas inversiones es necesario tratar de establecer cuáles son los factores de localización que tienen una mayor incidencia en las decisiones relativas a la elección del lugar de implantación de las distintas actividades directa o indirectamente relacionadas con la producción industrial y de servicios; ello implica tres niveles de análisis, el de las funciones de comando de las empresas respectivas, las actividades productoras de servicios y las industriales:

\section{Factores de localización para el comando del nuevo poder económico}

El hecho de que las grandes empresas que conforman la cúpula del nuevo poder económico, por lo general, están organizadas en red, implica que en la medida que su estructura contempla una organización económica territorialmente dispersa, ellas necesitan de una dirección y una coordinación centralizada, a fin de asegurar un manejo eficiente de su funcionamiento en el contexto de la economía global. Como afirma Sassen (1997: 50) "para permitirles a esas firmas globales ser competitivas en el mercado mundial, la proximidad física de sus centros de decisión con los expertos y la concentración de éstos en un espacio geográfico restringido juegan [...] un papel aún más importante que en el pasado". De esta manera, al decidir sobre la localización de sus sedes corporativas y de sus diversas funciones de comando (dirección, gerencia, planificación, gestión estratégica, etc.), tienden a privilegiar determinados puntos neurálgicos de la geografía global, al mismo tiempo que despliegan, hacia otros lugares, numerosos procesos y subprocesos productivos, conforme a estrategias en las que coexisten en su organización (nacional e internacional) la centralización y la descentralización. Básicamente, lo que otorga a determinados lugares esa condición de puntos neurálgicos sería la disponibilidad de:

a) mejores y más expeditos sistemas de comunicaciones, capaces de permitir contactos cotidianos fluídos con empresas relacionadas, que se encuentran ubicadas en distintos lugares del entorno global (aeropuerto internacional de primer nivel con amplia oferta de vuelos, red integrada de comunicaciones con el exterior, etc.); 
b) lo que se podría denominar como la "presencia del otro", esto es, la proximidad física de otras empresas de equivalente rango jerárquico, ya que para las cúpulas de estas empresas es un importante handicap el elegir una localización distante del lugar donde se concentra la mayoría de dichas empresas;

c) diversos tipos de servicios altamente especializados, cuyo producto las empresas prefieren adquirir externamente (servicios y analistas financieros, mercado de valores, expertos especializados en áreas de asesoría técnica, jurídica y publicidad, servicios médicos especializados de alto nivel, etc.) y,

d) finalmente, y no menos importante, las condiciones para una amplia y fluída comunicación directa cotidiana, formal e informal, entre las personas que desarrollan las tareas más creativas e innovadoras del nuevo aparato productivo, de manera de potenciar lo que Reich denomina los "beneficios creativos de la proximidad" (Reich, 1991: 236).

Dado que solamente es posible encontrar la conjunción de todos estos aspectos en las áreas metropolitanas principales (AMP) de cada país, es obvio que el comando de las grandes empresas nacionales y multinacionales que operan en ese ámbito, en especial aquéllas con mayor articulación con la economía global, tienda a localizarse mayoritariamente en aglomeraciones de este tipo. Dado que tanto la presencia como las decisiones y acciones de estos comandos pueden generar importantes efectos de arrastre sobre otras actividades productivas del entorno respectivo, su localización en estos lugares puede contribuir a intensificar la recuperación del crecimiento metropolitano.

\section{Factores de localización para el sector servicios}

En una economía en la que se generalizó la organización en red de las empresas y donde la externalización de las actividades de servicio adquirió creciente importancia, los servicios a la producción se situaron como uno de los componentes básicos del sector. Tanto para este tipo de servicios, como para los restantes servicios avanzados, se observó en los últimos años una fuerte tendencia a su concentración

metropolitana (Bailly, 1994). A este respecto, también se llegó a la conclusión de que las nuevas tecnologías de la información, contribuyen más a la centralización de los servicios que a su descentralización.

Es así, que se ha podido comprobar que ellos "permanecen concentrados en las áreas metropolitanas y a menudo en las ciudades más grandes, donde se benefician de importantes economías externas" (Bailly y Coffey, 1994: 227). Al respecto, estos autores afirman que los tres factores que explican en mayor grado la tendencia a la localización metropolitana de los servicios a la producción son:

a) la necesidad de mano de obra calificada, debido a que numerosos servicios dependen de la competencia de sus empleados y de la calidad de las relaciones interpersonales;

b) la necesidad de proximidad de creadores de conocimientos, de información y de técnicas, en especial porque los servicios a la producción deben desarrollar vínculos estrechos con otros productores de servicios que les son complementarios;

c) la proximidad del mercado, por lo que este tipo de servicios tienden a localizarse en la proximidad directa de las sedes sociales y las oficinas nacionales de las empresas, dado que es en éstas donde se adoptan la mayor parte de las decisiones de compras (Bailly y Coffey, idem).

Obviamente, es en las grandes áreas metropolitanas donde es posible encontrar la mejor y más amplia disponibilidad conjunta de estos factores, tanto en el plano internacional como nacional; ello las ha convertido en los lugares preferidos para la localización de este tipo de servicios, lo que ha redundado en un fuerte estímulo para la recuperación de las tendencias a la concentración metropolitana.

\section{Factores de localización para las nuevas industrias}

En lo que concierne a la industria, por el contrario a lo que anticiparon muchos analistas, solamente se observa cierta dispersión territorial selectiva, pese a las posibilidades que, en ese sentido, ofrecen 
las nuevas tecnologías de la información. ${ }^{10} \mathrm{Si}$ bien es cierto, que muchos procesos manufactureros se han dispersado hacia otros lugares de cada territorio nacional, ya sea para ubicarse en la proximidad de los recursos naturales o de mano de obra barata, o para aprovechar políticas locales de estímulo, la mayor parte de las actividades más dinámicas e innovadoras han mostrado una clara preferencia locacional por las AMP. No se trata, por tanto, de un renacimiento de las tendencias a la concentración territorial generalizada que caracterizó a la industria sustitutiva latinọamericana del periodo anterior, sino de un comportamiento diferenciado que afecta en forma desigual a distintos segmentos del nuevo aparato manufacturero. Comodestacan Caravaca y Méndez, (1992: 21) “[...] lo que se plantea ahora es la idea de que la gran ciudad 'pasa de una fase de crecimiento extensivo a una fase de crecimiento intensivo', en la que se refuerza la especialización en actividades intensivas en capital o conocimiento, tanto en la industria como en el terciario avanzado".

Este nuevo comportamiento locacional, puede explicarse teniendo en cuenta las condiciones generales de incertidumbre $y$ riesgo que se han intensificado en el escenario posfordista, donde la competitividad de cada empresa y de cada producto se constituye en un requisito para su supervivencia. En este contexto, la elección de una localización adecuada constituye un crucial aspecto adicional, en una gestión que tiene como uno de sus objetivos básicos, minimizar la incertidumbre y los riesgos, con base en estrategias en las que se combina la búsqueda de flexibilidad y de seguridades. En esa situación, y más allá de la atractividad del-entorno general, la industria especialmente la más moderna e innovadora - tiende a orientarse hacia los lugares que ofrecen ventajas en términos de mercados de trabajo, mercados para sus productos, contingentes de talento e innovación, complejidad de los tejidos industriales, infraestructura (especialmente en el área de las comunicaciones) y disponibilidad de servicios avanzados especializados, ventajas que, en su conjunto, solamente se pueden encontrar en las AMP preexistentes. En este sentido, Veltz (1996: 238) destaca: “[...] la dimensión metropolitana ofrece sobre todo una garantía frente a lo imprevisto y a lo imprevisible que parece muy superior a aquélla de otros tipos de territorio".

${ }^{10}$ En este sentido, Ascher, (1995:153) afirma que "[...] las nuevas tecnologías de transportes y de comunicaciones participan en las recomposiciones de los espacios urbanos rurales, pero no engendran una dispersión generalizada de los hombres y de las actividades"
En síntesis, los avances de los procesos de reestructuración y modernización capitalista -acompañados por los consecuentes procesos de desruralización y urbanización de la economía y el empleo- han llevado a la localización en las AMP de numerosas actividades secundarias y terciarias y, en particular, de la mayoría de las más representativas y dinámicas del nuevo aparato productivo posfordista. Como resultado de estos procesos ha comenzado a configurarse un nuevo mapa mundial en el que se señala que "[...] los impulsos dados al sistema mundo provienen de un cierto número de centros desde donde parten las iniciativas, donde nacen y son desarrolladas las innovaciones. Centros que son los emisores de órdenes, lugares de tratamiento, explotación y difusión de la información y donde, sobre una extensión restringida operan, en sinergía, las sedes de las principales empresas mundiales" (Dollfus, 1994: 22). Es bajo los dictados de esta lógica, que durante los últimos años el crecimiento metropolitano ha recuperado su dinamismo secular, pero basado ahora en un nuevo tipo de economía y funciones urbanas y en una nueva modalidad de expansión física.

El hecho de que el origen y el destino de los flujos de mayor relevancia, de la nueva dinámica económica posfordista, se ubique en un número limitado de grandes aglomeraciones, tiene una fuerte incidencia en una configuración territorial donde lo que más se destaca es la coexistencia de grandes manchas urbanas metropolitanas con grandes espacios semivacíos. En una situación en la que se contraponen las tendencias a la desruralización y a la urbanización de la economía y del empleo, la estructura territorial emergente, más que con base en regiones, está vertebrada en torno a un conjunto de metrópolis-regiones, donde se localizan los nodos principales de la respectiva red de flujos.

\section{Concentración productiva metropolitana y mercados urbanos} de trabajo en América Latina

En América Latina, desde los orígenes de la formación de las respectivas economías nacionales, las ciudades principales se constituyeron en los focos de irradiación, tanto para los respectivos procesos de integración económico-territorial, como para su articulación con la economía-mundo. Sin embargo, la plena consolidación de estas ciudades solamente llegó cuando se intensificó la urbanización de la economía bajo los impulsos de la industrialización sustitutiva, oportunidad en la que aumentó rápidamente su nivel de concen- 
tración productiva y demográfica. Fue en ese momento, cuando en los países más industrializados de la región comenzaron a desencadenarse incontrolables procesos de expansión metropolitana, entre los que se destacan especialmente los casos de la ciudad de México, São Paulo, Río de Janeiro, Buenos Aires, Bogotá, Santiago, Caracas y Lima.

Con la generalización de la aplicación de estrategias de apertura externa durante los últimos años, algunas de estas AMP se constituyeron en los lugares desde donde comenzaron a establecerse los nexos entre las actividades de cada país y las de las redes globales de comunicaciones, financieras, comerciales, productivas, culturales, etcétera. Al pasar a desempeñar el papel de nodo de articulación de las respectivas economías nacionales con el sistema global, se inició un cambio significativo en las funciones que estas AMP habían asumido en el periodo anterior, más relacionadas con la vertebración de sus respectivos espacios internos. A partir de ese momento, las AMP inician un proceso de modernización, compatible con la idea de que "las metrópolis modernas no son más sistemas autocentrados, sino potentes entrecruzamientos de redes múltiples" (Veltz, 1997: 61).

Ahora bien, esta transformación, en virtud de la que algunas AMP latinoamericanas han tendido a ubicarse como nodos de la red mundial de ciudades, adquiere relevancia únicamente en aquellos países en los que su nivel de reestructuración, les ha permitido iniciar un efectivo camino de modernización stricto sensu capitalista, sustentado en una estructura industrial posfordista y/o en una amplia terciarización de su economía. En definitiva, una transformación de esta naturaleza no depende tanto del número de grandes industrias existentes en un país, sino del tipo y de las características de las mismas, así como de la intensidad de los nexos, directos o indirectos, que ellas hayan podido establecer con las redes empresariales y productivas que se despliegan en el ámbito global. De igual forma, tampoco el tamaño de una metrópoli principal puede considerarse como un indicador de su grado de inserción en la red global de ciudades, puesto que esto está condicionado por las funciones que ella pueda haber asumido.

Por lo tanto, las tendencias a la recuperación del crecimiento metropolitano, a que hace referencia este trabajo, solamente podrán apreciarse en su verdadera magnitud a medida que en estos países avancen los procesos de recuperación y modernización económica compatible con la nueva dinámica globalizada. En este sentido, Azzoni (1995: 301-2) suministra un importante elemento de juicio a este respecto cuando - al cuestionar una posible desmetropolización para el caso brasileño- afirma que "la deseada recuperación económica del país ciertamente significará la ampliación de la producción, de la investigación y del comercio en las áreas más dinámicas, necesariamente vinculadas al área económica tradicional [áreas metropolitanas y grandes ciudades] de acumulación de capital”. De donde puede inferirse que los juicios sobre una mayor o menor dispersión territorial de la producción y de la población, antes que esos procesos se materialicen, sólo pueden interpretarse como transformaciones coyunturales, no generalizables como rasgos inherentes a la nueva fase del desarrollo capitalista posfordista.

A este respecto, es necesario tener presente que los procesos de reestructuración y modernización productiva, así como los de terciarización y urbanización, aún se encuentran en una fase intermedia en la mayoría de los países más industrializados de la región. Así, por ejemplo, el análisis de la estructura sectorial del empleo (Cuadro 1) en estos países, permite comprobar que muchos de ellos todavía cuentan con un elevado porcentaje de sus trabajadores ocupados en el sector agrícola y con una relativamente baja ocupación en el sector terciario, donde seguramente una parte importante corresponde a los servicios tradicionales de baja productividad (Cuadro 1).

Cuadro 1

Porcentaje de la población empleada en edad activa por sectores, 1960-1990

\begin{tabular}{||l|c|c|c|c|c|c||}
\hline & \multicolumn{3}{|c|}{1960} & \multicolumn{3}{c|}{1990} \\
\cline { 2 - 7 } & Agricul. & Indust. & Servicios & Agricul. & Indust. & Servicios \\
\hline Argentina & 21 & 34 & 45 & 12 & 32 & 55 \\
\hline Brasil & 55 & 17 & 28 & 23 & 23 & 54 \\
\hline Chile & 30 & 30 & 39 & 19 & 25 & 56 \\
\hline Colombia & 52 & 19 & 29 & 27 & 23 & 50 \\
\hline México & 55 & 19 & 25 & 28 & 24 & 48 \\
\hline Perú & 52 & 20 & 28 & 36 & 18 & 46 \\
\hline Uruguay & 21 & 29 & 50 & 14 & 27 & 59 \\
\hline Venezuela & 33 & 22 & 44 & 12 & 27 & 61 \\
\hline \hline
\end{tabular}

Fuente: PNUD (1996). Informe sobre Desarrollo Humano 1997. Ediciones Mundi Prensa, Madrid

Por otra parte, algunos indicadores sobre el nivel de desarrollo alcanzado (índice de Desarrollo Humano, PIB real per cápita, grado de urbanización, participación en las 500 mayores empresas, etc.), también muestran las disparidades existentes en el grado de avance de 
la recuperación económica de estos países (Cuadro 2). Complementariamente, la participación por país en las 90 empresas latinoamericanas de mayor patrimonio bursátil que transan acciones vía American Deposits Receipts (ADR) en la Bolsa de Nueva York, constituye un indicador sobre las importantes diferencias existentes en el grado de articulación de cada uno de ellos en las redes globales.

Cuadro 2

Indicadores de desarrollo e inserción

\begin{tabular}{||l|c|c|c|c|c|c||}
\hline & $\begin{array}{c}\text { 1. Indice } \\
\text { DH }\end{array}$ & $\begin{array}{c}\text { 2. PIB Real } \\
\text { pc }\end{array}$ & $\begin{array}{c}\text { 3. Grado } \\
\text { Urbaniz. }\end{array}$ & $\begin{array}{c}\text { 4. Part. 500 } \\
\text { Empresas }\end{array}$ & $\begin{array}{c}\text { 5. Part. } \\
\text { 90 ADR }\end{array}$ & $\begin{array}{c}\text { 6. Tamaño } \\
\text { Mercado }\end{array}$ \\
\hline Argentina & 0,884 & 8.937 & 85.9 & 73 & 16 & 194.6 \\
\hline Brasil & 0,783 & 5.382 & 73.9 & 243 & 21 & 438.7 \\
\hline Chile & 0.891 & 9.129 & 84.6 & 32 & 22 & 44.4 \\
\hline Colombia & 0.848 & 6.107 & 69.5 & 27 & - & 55,8 \\
\hline México & 0.853 & 7.384 & 72.7 & 102 & 21 & 175.0 \\
\hline Perú & 0.717 & 3.645 & 70.0 & 5 & 5 & 43.2 \\
\hline Uruguay & 0.883 & 6,752 & 88.8 & 3 & - & 13.9 \\
\hline Venezuela & 0.861 & 8.120 & 87.5 & 13 & 5 & 53.9 \\
\hline
\end{tabular}

Fuentes: 1, 2 y 3: PNUD (1996); 4 y 5: América Economia, Santiago, noviembre 1997; 6: STRATEGY RESEARCH CORPORATION, Latin American Market Planning Report (América Economia, diciembre 1997).

1) Índice de Desarrollo Humano 1997; 2) PB real per cápita; 3) Grado de urbanización; 4) Participación en las 500 mayores empresas de América Latina (América-Economía); 5) Participación en los 90 ADR latinoamericanos de mayor patrimonio bursátil; 6) Poder de compra en América Latina en 1996 en USS miles do millones.

En esta situación, sin embargo, ya se han comprobado avances claros en la marcha hacia la recuperación del crecimiento metropolitano y, en particular, del área metropolitana de mayor importancia de cada país, para el caso de las más importantes economías emergentes latinoamericanas. A este respecto, se pueden mencionar diversos estudios que, desde perspectivas diferentes, consignan síntomas de este tipo de evolución para países como Argentina (Blanco, 1996; Ciccolella, 1997), Colombia (Cuervo, 1997; Urrea Giraldo, 1997), Chile (de Mattos, 1996; Riffo Pérez y Silva, 1998), México (Delgado, 1992; Hiernaux, 1996), entre otros.

A partir de estos cambios, es previsible que las AMP de los países que logren intensificar su recuperación económica en la dirección señalada, podrán continuar intensificando sus nexos con la red global de flujos interurbanos $\mathrm{y}$, por lo tanto, sus procesos de expansión. Si se tiene en cuenta, que la mayoría de las economías emergentes latinoamericanas no disponen de una pluralidad delugares dotados de los atributos requeridos para acoger a las actividades de punta del aparato productivo posfordista, no hay duda que serán las más importantes de ellas las que podrán hacerlo. Al asumir este papel pasarán a consolidarse como sede principal de un conjunto de funciones y actividades que, a su escala, corresponden a las que a nivel mundial han sido señaladas como atributos de una ciudad global (Sassen, 1991), tales como:

a) las funciones de dirección, gestión, coordinación y control de las principales estructuras empresariales (conglomerados económicos y financieros, empresas multinacionales grandes empresas) que comandan la dinámica de acumulación, así como la articulación con la economía global;

b) las actividades básicas del terciario avanzado como servicios financieros (en especial la bolsa de valores), servicios al productor, servicios educacionales de punta, servicios vinculados a los productos y actividades globales, actividades culturales de mayor nivel, etcétera;

c) el núcleo más dinámico e innovador de la nueva industria, $y$

d) el mercado para la parte más relevante de los productos globales.

Al concentrar la mayor parte de estas actividades, estas aglomeraciones podrán afirmarse en su calidad de nodos secundarios de la red de ciudades estructurada a escala global. El aumento de su capacidad para asumir las funciones que intensifican su internacionalización, les permitirá potenciar su imbricación en esa red de ciudades y, con ello, mejorar su nivel de inserción y la posición de la respectiva economía nacional en la nueva dinámica globalizada.

Aun cuando en algunos de estos países (especialmente Brasil, México y Colombia), los fenómenos de expansión involucran a varias áreas metropolitanas, es en general en una de ellas donde se concentra la mayor parte de las cúpulas de las principales funciones empresariales y financieras. Tal es el caso de las bolsas de valores, cuya 
máxima expresión se encuentra, por lo general, localizada en las AMP de mayor gravitación económica de cada país, ${ }^{11}$ pese a que allí pueda existir más de una entidad de esta naturaleza. Por su parte, la presencia de la principal bolsa de valores en estas metrópolis, se complementa invariablemente con la localización en su entorno de las cabezas de resto del aparato financiero (bancos, fondos previsionales, seguros, etcétera). También es en ese lugar, donde en la mayor parte de los casos, se concentra la cabeza de los organismos corporativos de la empresa privada, las representaciones diplomáticas y comerciales del resto del mundo y desde donde se realiza la mayoría de los enlaces comunicacionales y aéreos internacionales.

Por consiguiente, la localización en estas metrópolis de dicho conjunto de funciones y actividades determina que sea en ellas donde se estructuren los mercados de trabajo de mayor envergadura, más diversificados y más dinámicos de cada espacio nacional. A partir de las actividades acumuladas en cada uno de estos subsistemas a lo largo de su historia - y de las externalidades que desde allí se propagan- se ha ido generando un set extraordinariamente diversificado y complejo de actividades que es, justamente, lo que otorga su especificidad a los mercados metropolitanos de trabajo.

Sumadas las nuevas funciones y ocupaciones, inherentes a su progresiva terciarización, en estos mercados coexiste, imbricado po innumerables canales, una multitud de tipos de trabajo, comprendiendo desde aquellos que reciben las más elevadas remuneraciones de su ámbito nacional hasta el número creciente de ocupaciones marginales y/o periféricas, entre las que se incluye una variada gama de empleos precarios, con remuneraciones paupérrimas. La caracterización de un mercado de trabajo metropolitano, en el sentido de que "es más una colección de segmentos que un mercado lineal y homogéneo" (Perulli, 1992: 38), resulta pertinente para el caso de las economías emergentes latinoamericanas. En cualquier caso, la existencia de esta diversidad de segmentos laborales es lo que les permite a estas aglomeraciones, ofrecer posibilidades de supervivencia sin parangón en cada espacio nacional y mantener la continuidad de su potencial de atracción.

¿Cuáles podrían ser los principales efectos de esta situación sobre los movimientos de población? Dado que en el escenario posfordista, la amenaza de la desocupación ha cobrado una presencia

${ }^{11}$ Los índices Merval de la Bolsa de Buenos Aires, Bovespa de São Paulo, Índice Genera de Ciudad de México, IPSA de Santiago, etc., son los considerados realmente relevantes par apreciar la situacion de los mercados financieros de sus respectivos paises, independientemente del hecho de que en otras ciudades de los mismos funcionen otras bolsas de valores.

más atemorizante, puede preverse que la fuerza de trabajo (en especial, la de mayor calificación) muestre preferencia por los mercados de trabajo más amplios, consolidados y dinámicos, donde la posibilidad de obtener empleo aparecerá como más promisoria. De tal forma que es muy probable que las AMP continúen siendo uno de los destinos preferidos de los movimientos de población. Así, de esta manera, en el plano internacional los movimientos de población se orientarán principalmente hacia los países ricos y en el plano nacional la población tenderá a desplazarse desde los mercados regionales o locales estancados o deprimidos hacia los que muestren mayor crecimiento y dinamismo $\mathrm{y}$, en particular, hacia los más grandes $\mathrm{y}$ diversificados. En este contexto, las AMP deberían continuar ejerciendo su fuerza de atracción.

Por otra parte, es previsible que en los países en los que los procesos de modernización redunden en mayor crecimiento económico, simultáneamente con la expansión de las AMP, habrá de continuar el crecimiento de un número importante de ciudados de tamaño medio, en función de la dinamización de sus respectivos mercados de trabajo, a base del desarrollo de ciertas actividades productivas, generalmente vinculadas a ventajas comparativas vis-a-vis con los mercados externos en el marco de la globalización, muchas veces relacionadas con la utilización de recursos naturales y/o de mano de obra barata. Además, también puede preverse el rápido crecimiento de un conjunto de ciudades de tamaño intermedio, ubicadas en las áreas de gravitación directa de las áreas metropolitanas de mayor expansión física; sin embargo, considerando las características que, en la actualidad ha asumido la expansión metropolitana, surgen dudas acerca de si en estos casos, efectivamente, puede hablarse de una ciudad intermedia o, más apropiadamente, de un componente suburbano de la metrópoli.

En contraposición, bajo las condiciones impuestas por la liberalización económica y la desregulación, también se encuentran aquellas que Ciccolella (1996) denomina como regiones (o ciudades) "no rentables", donde a falta de algún tipo concreto de ventaja comparativa, no hay condiciones para atraer inversiones, generar puestos de trabajo y, por ende, establecer condiciones para mejorar las condiciones de vida de sus habitantes. Forman parte del contingente de lo que, algunos autores, han denominado como las regiones o ciudades perdedoras. En este cuadro general, hay suficiente evidencia empírica sobre las tendencias que se están desplegando bajo la dinámica de la reestructuración que no autorizan a inferir, como se ha venido haciendo, que se esté produciendo un crecimiento generalizado 
de las ciudades medias; en el mejor de los casos se podría hablar del crecimiento y la prosperidad de algunas de ellas, vinculadas a un número limitado de procesos o áreas productivas dinámicas.

\section{Expansión productivo-demográfica, estrategias privadas y metropolización expandida}

¿Cómose materializan territorialmente estos procesos de crecimiento metropolitano? Quizás el rasgo más relevante de la expansión territorial de las AMP de la época de la globalización y de la desregulación, sea su incontrolable tendencia a la suburbanización y/o periurbanización, a partir de los núcleos urbanos originales, en un proceso en el que la mancha metropolitana se expande en forma incesante, ocupando las áreas rurales que encuentra a su paso y desbordando los límites urbanos definidos en el momento anterior. ${ }^{12}$ Así, el área urbana heredada del periodo desarrollista, cuyos límites aparecían dibujados en forma relativamente más precisa y nítida, ha ido dando paso a una metrópoli-región ${ }^{13}$ policéntrica, que en su persistente expansión se va configurado como un verdadero archipiélago urbano (Ferrao, 1992; Dollfus, 1994) de fronteras difusas.

Esta modalidad de expansión urbana que comienza a predominar en el mundo entero, adquiere manifestaciones específicas en las ciudades de las economías emergentes periféricas, donde diversos centros urbanos, hasta entonces autónomos, son absorbidos por el derrame de la mancha urbana o, simplemente, incorporados sistémicamente a la dinámica metropolitana. Así, en casos como Sao Paulo ${ }^{14}$ y ciudad de México, el archipiélago resultante se extiende

${ }^{12}$ Una reciente investigación sobre doce grandes "city-regions", entre las que se incluyeron dos latinoamericanas (Sao Paulo y Santiago), concluyó que: "en todas estas ciudades, el modelo dos latinoamericanas (Sao Paulo y Santiago), concluyb que: "en todas estas ciudades, el modelo cen interiores adyacentes hacia distantes espacios abiertos y tierra agricola" (Lincoln Institute,
1996: 3).

${ }^{13}$ Diversas denominaciones han estado siendo utilizadas para denominar a este tipo de configuración urbana, tales como area metropolitana, region metropolitana, ciudad-región, región-urbana, megalópolis, megápolis, metápolis (Ascher, 1995), ciudad global (Sassen, 1991) aún cuando esta última se utiliza con una connotación específica.

${ }^{14}$ En el caso de Brasil, dada su dimensión continental, esta modalidad de concentración se manifiesta dentro de un área significativamente mayor: "[...] a pesar de los efectos de frontera arríla del Centro-Oeste y de la frontera mineral del Norte y de los incentivos ficcles para las repiones Norte y Nordeste, los cuales ayudan a explicar el crecimiento de

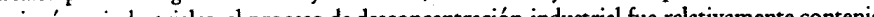

tentacularmente por una enorme extensión en la que terminan siendo afectadas otras áreas metropolitanas cercanas. En estas estructura predominantemente urbanas, suburbanizadas y policéntricas, el modelo de ciudad de corte europeo, que se había constituido en el referente obligado en los momentos de mayor esplendor de la ciudad latinoamericana, ha ido cediendo paso a un modelo en el que Los Ángeles aparece como paradigma.

En cualquier caso, esta modalidad de expansión urbana no puede considerarse como un fenómeno enteramente nuevo, sino como la acentuación $-y$, hasta cierto punto, como la lógica y previsible culminación-de un rasgo inherente a la urbanización capitalista, que ya había comenzado a perfilarse en el periodo desarrollista. Lo que aparece como específico de este periodo es que la concentración productiva y demográfica cristaliza en una metropolizaciónexpandida, en la medida que un importante conjunto de procesos productivos, en especial los más tradicionales, así como también la población, ya no requiere concentrarse en un área compacta; sin embargo, un número significativo de dichos procesos requiere de una razonable proximidad entre sí y, al mismo tiempo, con el lugar donde se encuentran las mayores economías de aglomeración, ${ }^{15}$ todo lo cual augura la continuidad del crecimiento y la expansión de estos lugares.

¿Qué es lo que ha permitido y estimulado esta modalidad de concentración expandida? Básicamente, la imbricación de dos tipos de factores, unos de naturaleza tecnológica y otros de orden socioeconómico. En lo que respecta a los factores de tipo tecnológico, importa destacar la generalización del uso de tres tipos de productos asociados a la evolución del nuevo paradigma científico-técnico, cuya difusión ha sido una consecuencia natural del avance de la globalización y la desregulación:

a) el automóvil y los diferentes tipos de transporte automo tor. El vertiginoso aumento de la tasa de motoriza-

dentro de la Región Centro-Sur, en un gran polígono que va desde la región central de Minas Gerais hasta el nordeste de Rio Grande do Sul, dentro del cual están 54 de las 76 áreas industriales con más de 10.000 personas ocupadas en 1980, sin considerar 5 áreas del Estado de Río de Janeiro. Este fenómeno está relacionado con la fuerza del campo aglomerativo de São Paulo." (Campolina Diniz y Crocco, 1995: 10).

is Como afirma Peter Hall, "[...] las economías de aglomeración no han perdido nada de su importancia, y [...] ellas continúan jugando en favor de las grandes zonas metropolitanas no obstante los factores externos negativos que constituyen claramente los embotellamientos y la polución". (Hall, 1996:22). 
ción registrado en estos países y, en particular, en sus regiones metropolitanas, indica la generalización de su utilización familiar e individual; esto ha generado una demanda creciente por una infraestructura para su mejor utilización, con lo que las vías y carreteras y, en especial, las autopistas urbanas, se han constituido en los ejes que guían la expansión urbana, de lo que, frecuentemente, resulta una morfología metropolitana de tipo tentacular;

b) las nuevas tecnologias de la información y la comunicación, cuya adopción generalizada, al tiempo que han reducido significativamente la gravitación de la distancia como un factor limitante para la localización de las empresas y las familias, también han estimulado un aumento progresivo del trabajo realizado en el lugar de residencia;

c) La televisión, tanto abierta como por cable y satelital, cuya explosiva difusión, en todos los sectores sociales, ha favorecido un mayor afincamiento cotidiano en hogares situados a distancias relativamente mayores que las que prevalecían en la ciudad más concentrada del pasado.

En lo que respecta a los factores de orden socioeconómico, es importante destacar que lo que la liberalización económica ha logrado, en esencia, ha sido despejar el camino para la afirmación de una lógica estrictamente capitalista en la producción y la reproducción metropolitana, al permitir una operación desregulada (o, en el mejor de los casos, escasamente regulada) de dos tipos de estrategias sociales:

a) Las estrategias empresariales, que utilizan el espacio metropolitano para el desarrollo de un tipo específico de actividad productiva urbana, vinculada a la construcción civil y a los negocios inmobiliarios. Esto resulta del hecho de que estas metrópolis-región, en tanto receptoras de la parte más moderna y dinámica del aparato productivo del respectivo ámbito nacional, configuran un medio privilegiado e insustituible para la valorización privada del capital. En efecto, su continuada expansión genera ilimitadas oportuni- dades para el crecimiento de actividades directamente ligadas a los negocios inmobiliarios, en los que la renta de la tierra impulsa a la creación, por una parte, de áreas de concentración de actividades empresariales, con el consecuente aumento de la verticalización de sectores de la ciudad y, por otra, de nuevas áreas residenciales, con fuertes efectos en la expansión de los límites de la ciudad y en la disminución de la densidad urbana.

b) Las estrategias individuales o familiares de un cúmulo de actores que, en función de preferencias por la vivienda unifamiliar, desbordan reiteradamente las diversas disposiciones establecidas, con el propósito de regular el crecimiento y el funcionamiento urbanos. Por una parte, las estrategias habitacionales de los sectores más solventes que, en buena parte de los casos, responden al deseo de evadirse de diversos problemas, tienden a agudizarse en la parte central de estas AMP (contaminación, congestión, delincuencia, etc.) y buscan refugio en sitios escogidos de su periferia inmediata. Con ello, sin embargo, se intensificará la suburbanización y, de esta forma, se estimula la continuidad del fenómeno urbano-territorial del que se quiere escapar. Por otro lado, los sectores desposeídos, en lucha permanente por lugares de residencia, tienden a ocupar tierras marginales, muchas veces en forma ilegal, donde generalmente proliferan viviendas precarias y sin servicios, que también contribuyen a empujar la frontera urbana, a pesar de hacerse en otras direcciones.

Puede afirmarse entonces, que los negocios inmobiliarios, con la complicidad activa de la población urbana, juegan un papel fundamental en las tendencias actuales de la expansión metropolitana por la vía de la suburbanización. La confluencia de estas estrategias tiene como resultado una metrópoli-región que se construye y reconstruye, que se configura y reconfigura cotidiana y caóticamente, por lo que "lejos de ser un proyecto diseñado y controlado por el hombre, se ha convertido en una realidad que escapa a su control" (Naredo, 1994:234). 
Habida cuenta del alcance de las estrategias, tanto empresariales como familiares, que producen espacio metropolitano, es lógico inferir que cualquier intento por regular el funcionamiento de estas ciudades y atenuar su expansión exigiría un esfuerzo deliberado, orientado a controlar a un sinnúmero de actores y a restringir ( $y / o$ a impedir) una infinidad de negocios. Esto, obviamente, además de un enorme poder político e ideológico, requeriría de un manejo altamente autoritario. De allí que las perspectivas de una gestión urbana racional y efectiva, aparezcan cada día más lejanas, cuando menos mientras perduren los criterios por los que actualmente se rige la gestión pública.

Santiago de Chile, octubre 1997/febrero 1998

\section{Bibliografía}

Ascher, François (1995), "Dynamiques métropolitaines et enjeux socio-politiques", Futur Antérieur, París, núm. 29.

Azzoni, Carlos Roberto (1995), "Formaçao socio-espacial metropolitana: novas tendências ou novas evidências?" , en: Maria Flora Gonçalves (Org.), O Novo Brasil Urbano. Impasses. Dilemas. Perspectivas, Porto Alegre, Mercado Aberto.

Bailly, Antoine (1994), "Evolution des sistèmes de production et des localisations des activités de service. 25 ans au service des services”, en: Joël Bonamy y Nicole May (Orgs.), Services et mutations urbaines. Questionnements et perspectives, París, Economica.

- William J. Coffey (1994), “Localisation des services à la production et restucturations économiques", L'Espace Géographique, París, núm. 1.

Blanco, Jorge Oscar (1996), “Area Metropolitana de Buenos Aires: transformaciones territoriales en el marco de la globalización", Revista EURE, Santiago de Chile, vol. XXII, núm. 67, diciembre.

Campolina Diniz, Clelio y Marco Aurelio Croceo (1995), "Reestructuración productiva y nuevos distritos industriales en Brasil:

el nuevo mapa de la industria brasileña”, Revista de Estudios Regionales, Málaga, núm. 43, diciembre.

Caravaca, Inmaculada y Ricardo Méndez (1992), “Crisis y crecimiento de la industria metropolitana", Revista EURE, Santiago de Chile, vol. XVIII, num. 55, octubre.

CEPAL (1995), La inversión extranjera en América Latina y el Caribe, Santiago de Chile, Comisión Económica para América Latina y el Caribe.

Ciccolella, Pablo (1996), "Reestructuración global, transformaciones económicas en la Argentina y reterritorialización de la región metropolitana de Buenos Aires. Hacia una ciudad competitiva, globalizada y excluyente", Revista de Estudios Regionales, Málaga, núm. 43, septiembre-diciembre.

Chinitz,Benjamín y Thomas Moran (1996), "Comunications Technology and Settlement Patterns”, Landlines (Lincoln Institute of Land Policy), vol. 8, núm. 5, septiembre.

Coriat, Benjamín (1997), Los desafíos de la competitividad. Buenos Aires, Asociación Trabajo y Sociedad/Oficina de Publicaciones del CBC-Universidad de Buenos Aires.

Cuervo, Luis Mauricio y Josefina Gonzalez (1997), Industria y ciudades en la era de la mundialización. Un enfoque socioespacial, Bogotá, TM Editores.

Davidow, William H. y Michael S. Malone (1992), The Virtual Corporation. Structuring and Revitalizing the Corporation for the $21^{\text {st }}$ Century, New York, Harper Business.

Delgado, Javier (1992), “Tendencias megalopolitanas en la ciudad de México", en: CONAPO, La Zona Metropolitana de la Ciudad de México: situación actual y perspectivas demográficas y urbanas. México, CONAPO, diciembre.

De Mattos, Carlos A. (1996), "Avances de la globalización y nueva dinámica metropolitana: Santiago de Chile, 1975-1995”, Revista EURE, Santiago de Chile, vol. XXII, núm. 65. 
Dollfus, Olivier (1994), L'espace monde, París. Ed. Economica.

Dosi, Giovanni (1991), “Una reconsideración de las condiciones y modelos del desarrollo. Una perspectiva 'evolucionista' de la innovación, el comercio y el crecimiento", Pensamiento Iberoamericano, Madrid, núm. 20, julio-diciembre.

Ferrao, Joao (1992), Serviços e inovaçao. Novos caminbos para o desenvolvimento regional, Oeiras, Celta Editora.

Guellec, Dominique y Pierre Ralle (1995), Les nouvelles théories de la croissance, París, La Decouverte.

Hall, Peter (1996), “La ville planétaire”, Revue Internationale des Sciences Sociales, París, núm. 147, marzo.

Hatem, Fabrice (1995), Les multinationales de l'an 2000, París, Economica.

Hiernaux Nicolas, Daniel (1996), "Desigualdades sociales y exclusión en la reestructuración económica y territorial de México”, III Seminario Internacional Impactos Territoriales de los Procesos de Reestructuración, La Rábida, España, septiembre.

Kampetter, Werner (1995), "Fertilidad nacional, Estado-nación y sistema económico mundial”, Nueva Sociedad, Caracas, núm. 137, mayo-junio.

Krugman, Paul (1992), Geografía y comercio, Barcelona, Antoni Bosch Editor.

Lafay, Gérard (1996), Comprendre la mondialisation, París, Economica.

Lincoln Institut Of Land Policy (1996), "Global City Regions: Searching for Commond Ground", Landlines, vol.8, núm. 1, enero.

Lipietz, Alain y Danielle Leborgne (1988), "L'après fordisme et son espace”, Le Temps Modernes, París, num. 43, abril.
Marconnet, Isabelle y Philippe Fremeaux (1996), "La nouvelle geographie de l'investissement internationale", Alternatives Economiques, num. 133, enero.

Menzel, Ulrich (1995), "La revolución post industrial. Terciarización y desmaterialización de la economía”, $D+C$ Desarrollo $y$ Cooperación, Francfort, núm. 5.

Naredo, José Manuel (1994), “El funcionamiento de las ciudades y su incidencia en el territorio", Ciudad y Territorio/Estudios Territoriales, Madrid, Vol. II, núms. 100-101, verano-otoño.

Ohmae, Kenichi (1995), The End of the Nation State, The Free Press.

Perulli, Paolo (1992), Atlas metropolitano. El cambio social en las grandes ciudades, Madrid, Alianza Editorial, 1995.

PNUD (Programa de las Naciones Unidas para el Desarrollo) (1997), Informe sobre desarrollo bumano 1997, Madrid, Ediciones Mundi Prensa.

Porter, Michael E. (1990), La ventaja competitiva de las naciones, Buenos Aires, Javier Vergara Editor, 1991.

Reich, Robert (1991), The Work of Nations. Preparing Ourselves for $21^{\text {st }}$ Century Capitalism, Vintage Books, Nueva York, 1992.

Riffo Perez, Luis y Verónica Silva (1998), “Las tendencias locacionales de la industria en el marco de los procesos de reestructuración y globalización en Chile", en: Carlos De Mattos, Daniel Hiernaux y Darío Restrepo (Orgs.), Globalizacióny territorio, Santiago de Chile, Fondo de Cultura Económica.

Sassen, Saskia (1997), "Les villes jouent un rôle central dans la mondialisation", Alternatives Economiques, París, núm. 150, julioagosto.

(1991), The Global City. New York, London, Tokio, Princeton, Nueva Jersey, Princeton University Press. 